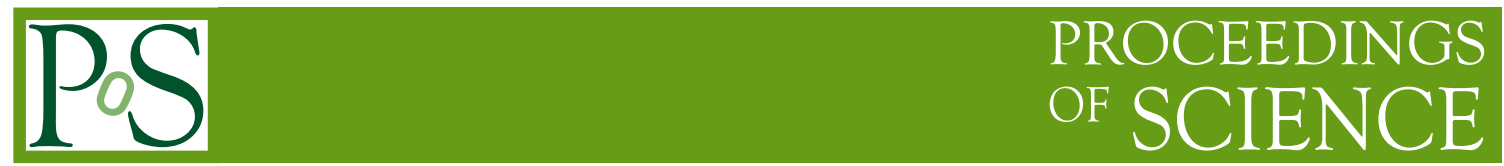

\title{
NNNLO results on top-quark pair production near threshold*
}

\section{Martin Beneke ${ }^{\dagger}$}

Institut für Theoretische Physik E, RWTH Aachen, D-52056 Aachen, Germany

E-mail: mbenekeATphysik.rwth-aachen.de

\section{Yuichiro Kiyo}

Institut für Theoretische Teilchenphysik, Universität Karlsruhe, D-76128 Karlsruhe, Germany

\section{Kurt Schuller}

Institut für Theoretische Physik E, RWTH Aachen, D-52056 Aachen, Germany

We present new results on the NNNLO top-antitop production cross section near threshold from potential and ultrasoft gluon corrections. The new non-logarithmic third-order terms are in the $10 \%$ range and lead to a significant reduction in the theoretical error.

8th International Symposium on Radiative Corrections

October 1-5, 2007

Florence, Italy

*preprint PITHA 08/03, TTP/08-04, SFB/CPP-08-07

† Speaker. 


\section{Introduction}

High-order perturbative calculations of non-relativistic heavy quark-antiquark systems are required for precise quark mass determinations from QCD sum rules or the lowest upsilon states (bottom quark mass) or the energy dependence of the threshold top-quark pair production cross section in $e^{+} e^{-}$collisions (top quark mass). Concerning the $t \bar{t}$ quark cross section the status is as follows: the NNLO calculations performed about ten years ago revealed a large uncertainty, up to $\pm 25 \%$, in the cross section in the resonance peak region [1,2]. Subsequent calculations that include a summation of logarithms of $\alpha_{s}$ find a much reduced scale dependence around $\pm(3-6) \%$ $[3,4]$. The main effect comes from the logarithms at the third order (NNNLO) rather than the all-order series [5]. Since at NNNLO the ultrasoft scale $m_{t} \alpha_{s}^{2} \sim 2 \mathrm{GeV}$ appears for the first time, a complete calculation of the (non-logarithmic) NNNLO correction is needed. Similar conclusions apply to bottomonium systems, with larger uncertainties.

Recently the NNNLO correction to the $S$-quarkonium wave-functions at the origin (corresponding to the residues of the poles of the heavy quark current spectral functions) from potential insertions and ultrasoft gluons have been completed $[6,7]$. The talk presented at this conference summarized these results together with NNNLO results on the full energy-dependent spectral function [8] relevant to the $t \bar{t}$ cross section. Since the combined result of [6, 7] has already been discussed in another proceedings article [9], we focus here on the full spectral function and the case of the top quark. That is, we consider the two-point function

$$
\left(q^{\mu} q^{v}-g^{\mu v} q^{2}\right) \Pi\left(q^{2}\right)=i \int d^{d} x e^{i q x}\left\langle\Omega\left|T\left(j^{\mu}(x) j^{v}(0)\right)\right| \Omega\right\rangle
$$

of the electromagnetic top-quark current $j^{\mu}=\bar{t} \gamma^{\mu} t$, choosing $q^{\mu}=\left(2 m_{t}+E, \overrightarrow{0}\right)$ with $m_{t}$ the pole mass of the top quark and $E$ of order of a few GeV. The width of the top quark is taken into account by simply letting $E \rightarrow E+i \Gamma_{t}$ become complex [10]. However, one should note that a fully consistent treatment of the top-quark decay beyond the NLO approximation requires the inclusion of many other electroweak effects that are not yet known.

\section{Remarks on the calculation}

After integrating out the hard and soft momentum scales the problem is reduced to the calculation of a non-relativistic correlation function $G(E)$ to third order in non-relativistic perturbation theory. The perturbations consist of potential insertions (instantaneous, spatially non-local operators) and ultrasoft gluon interactions with the top quarks. Since an infinite number of potential (Coulomb) gluons can be exchanged between the heavy quarks without parametric suppression, the free heavy quark-anti-quark propagators are promoted to the Green function of the Schrödinger operator $H_{0}=-\vec{\nabla}^{2} / m_{t}-\left(\alpha_{s} C_{F}\right) / r$ with the colour Coulomb potential $\left(C_{F}=4 / 3\right)$. Computing Feynman integrals with Coulomb Green functions while simultaneously regulating all divergences dimensionally to be consistent with fixed-order matching calculations is the main challenge of the NNNLO calculation. The third-order correction to $G(E)$ is composed of

$$
\delta_{3} G=-\left\langle 0\left|\hat{G}_{0} \delta V_{1} \hat{G}_{0} \delta V_{1} \hat{G}_{0} \delta V_{1} \hat{G}_{0}\right| 0\right\rangle+2\left\langle 0\left|\hat{G}_{0} \delta V_{1} \hat{G}_{0} \delta V_{2} \hat{G}_{0}\right| 0\right\rangle-\left\langle 0\left|\hat{G}_{0} \delta V_{3} \hat{G}_{0}\right| 0\right\rangle+\delta G_{\text {us }}
$$


where $\hat{G}_{0}=\left(H_{0}-E-i \varepsilon\right)^{-1},|0\rangle$ denotes a relative position eigenstate with eigenvalue $\mathbf{r}=0$, and $\delta V_{i}$ the $i$ th order perturbation potentials (see [6]). The contributions to $G(E)$ involving only higherorder corrections to the Coulomb potential have already been computed in [11] and are included in the following numerical result. The ultrasoft contribution is $(D=d-1)$

$$
\begin{aligned}
\delta G_{\mathrm{us}}= & (-i)\left(i g_{s}\right)^{2} C_{F} \int \frac{d^{d} k}{(2 \pi)^{d}} \frac{-i}{k^{2}}\left(\frac{k^{i} k^{j}}{k_{0}^{2}}-\delta^{i j}\right) \int \prod_{n=1}^{6} \frac{d^{D} p_{n}}{(2 \pi)^{D}} i \tilde{G}^{(1)}\left(p_{1}, p_{2} ; E\right) \\
& \times i\left[\frac{2 p_{3}^{i}}{m_{t}}(2 \pi)^{D} \delta^{(D)}\left(p_{3}-p_{2}\right)+\left(i g_{s}\right)^{2} \frac{C_{A}}{2} \frac{2\left(p_{2}-p_{3}\right)^{i}}{\left(p_{2}-p_{3}\right)^{4}}\right] i \tilde{G}^{(8)}\left(p_{3}, p_{4} ; E+k^{0}\right) \\
& \times i\left[-\frac{2 p_{4}^{j}}{m_{t}}(2 \pi)^{D} \delta^{(D)}\left(p_{4}-p_{5}\right)+\left(i g_{s}\right)^{2} \frac{C_{A}}{2} \frac{2\left(p_{4}-p_{5}\right)^{j}}{\left(p_{4}-p_{5}\right)^{4}}\right] i \tilde{G}^{(1)}\left(p_{5}, p_{6} ; E\right)
\end{aligned}
$$

with $\tilde{G}^{(1,8)}\left(p, p^{\prime} ; E\right)$ the colour-singlet/octet momentum-space Coulomb Green functions. In position space this expression simplifies to three instead of seven loop integrations and similar simplifications apply to the other terms in (2.1). However, the integrals are divergent and the $1 / \varepsilon$ poles must be extracted in momentum space. A further complication is that the Coulomb Green functions are not known in $D$ dimensions. The strategy therefore consists of identifying all divergent subgraphs, and to calculate them in $d$-dimensional momentum space. Then combine the result with the sub-divergence counterterms related to the renormalization of potentials and non-relativistic currents and perform the remaining integrations in three dimensions. Due to the incomplete treatment of finite width effects an over-all divergence $\alpha_{s}^{1,2} / \varepsilon \times \Gamma_{t}$ remains in the $t \bar{t}$ cross section, which is minimally subtracted in the result below.

\section{Size of logarithmic and non-logarithmic terms}

We consider the residue of the correlation function at the lowest-energy bound-state pole at $E_{1}=-m_{t}\left(\alpha_{S} C_{F}\right)^{2} / 4+\ldots$ to compare the new NNNLO non-logarithmic terms [6, 7] to the previously known logarithms $[12,13,14,15]$, since in this case a simple numerical result can be given. $Z_{1}$, defined by

$$
\Pi\left(q^{2}\right) \stackrel{E \rightarrow E_{1}}{=} \frac{3}{2 m_{t}^{2}} \frac{Z_{1}}{E_{1}-E-i \varepsilon},
$$

is related to the height of the cross section peak by $R_{\text {peak }} \approx 18 \pi e_{t}^{2} Z_{1} /\left(m_{t}^{2} \Gamma_{t}\right)$, so we expect similar conclusions to hold for the entire $t \bar{t}$ cross section. The NNNLO expression for $Z_{1}$ reads

$$
\begin{aligned}
Z_{1}= & \frac{\left(m_{t} \alpha_{s} C_{F}\right)^{3}}{8 \pi} \times\left(1+\alpha_{s}[-2.13+3.66 L]\right. \\
& +\alpha_{s}^{2}\left[8.38-7.26 \ln \alpha_{s}-13.40 L+8.93 L^{2}\right] \\
& +\alpha_{s}^{3}\left[11.01+[37.58]_{c_{3}, n_{f}}-9.79 \ln \alpha_{s}-16.35 \ln ^{2} \alpha_{s}\right. \\
& \left.\left.\quad+\left(53.17-44.27 \ln \alpha_{s}\right) L-48.18 L^{2}+18.17 L^{3}\right]\right) \\
& \quad \frac{\left(m_{t} \alpha_{s} C_{F}\right)^{3}}{8 \pi} \times\left(1-2.13 \alpha_{s}+22.64 \alpha_{s}^{2}+\left[-32.96+[37.58]_{c_{3}, n_{f}}\right] \alpha_{s}^{3}\right) .
\end{aligned}
$$


Since the scales $m_{t}, m_{t} \alpha_{s}$ and $m_{t} \alpha_{s}^{2}$ are all relevant here, there is an ambiguity in the representation of the logarithms. The above result uses $\alpha_{s} \equiv \alpha_{s}(\mu)$ and puts the explicit $\mu$-dependence into $L \equiv$ $\ln \mu /\left(m_{t} C_{F} \alpha_{s}\right)$. The NNNLO result is not yet complete: the constant 11.01 includes an estimate of the NNNLO correction to the Coulomb potential, $a_{3}=3840$ [16], and sets certain unknown $\mathscr{O}(\varepsilon)$ potential terms to zero. This is expected to have a minor effect [6]. More important is that only the $n_{f}$-parts of the third-order matching coefficient $c_{3}$ of the non-relativistic current $\psi^{\dagger} \sigma^{i} \chi$ are known [17], which turn out to be very large $\left([37.58]_{c_{3}, n_{f}}\right)$. In the following numerical results for the $t \bar{t}$ cross section we therefore consider two options, one where the constant part of $c_{3}$ is set to the known $n_{f}$ terms, the other where it is set to zero. (The logarithms are all known and always included.)

We observe (third and fourth line of (3.2)) that the typical size of non-logarithmic terms of individual third-order corrections (ultrasoft, non-Coulomb potentials, Wilson coefficient) is about $40 \alpha_{s}^{3} \approx 10 \%$ (>100\%) for toponium (bottomonium). However, large cancellations between individual terms and between logarithmic and non-logarithmic terms occur. Thus, to obtain a reliable third-order result the non-logarithmic terms are crucial and a final assessment needs the missing $n_{f}$-independent term in $c_{3}$. This is seen in the last line of (3.2), which shows the series for $\alpha_{s}=0.14$ where $L=0$. The large NNLO correction is evident. On the other hand, the third-order correction is not anomalously large, although the final coefficient will only be known when the term $[37.58]_{c_{3}, n_{f}}$ is replaced by the full result for $c_{3}$. The NNNLO result shows a strong reduction of the scale dependence compared to NNLO as discussed in [9], but the perturbative prediction becomes unstable for $\mu<20 \mathrm{GeV}$. A study of this problem for the Coulomb corrections, where a resummation of the perturbative series can be done by means of a numerical solution, has shown [11] that the perturbative prediction for $\mu>25 \mathrm{GeV}$ is close to the true result, hence we do not consider scales $\mu<25 \mathrm{GeV}$.

\section{Top-quark cross section}

We next discuss the $t \bar{t}$ production cross section in $e^{+} e^{-}$annihilation near threshold. More precisely, we consider the $R$-ratio

$$
R=\sigma_{t \bar{t} X} / \sigma_{0}=12 \pi e_{t}^{2} \operatorname{Im} \Pi\left(q^{2}\right) \quad\left(\sigma_{0}=4 \pi \alpha_{\mathrm{em}}^{2} /(3 s)\right),
$$

neglecting the axial-vector contribution from $Z$-exchange for the purpose of discussing the impact of the QCD NNNLO correction. The top quark pole mass should be avoided as an input parameter. Here we use the potential-subtracted mass [18] implemented as explained in [11]. The parameters for the cross section calculation are: $m_{t, \mathrm{PS}}(20 \mathrm{GeV})=175 \mathrm{GeV}, \Gamma_{t}=1.4 \mathrm{GeV}, \alpha_{s}\left(M_{Z}\right)=0.1189$.

The successive LO ... NNNLO approximations to $R$ are shown in Figure 1 (top panel). At $\mu=30 \mathrm{GeV}$ the size of the third-order correction is up to $10 \%$ depending on the assumption for $c_{3}$. When all known terms in $c_{3}$ are included the peak cross section is about $10 \%$ larger than in the renormalization-group improved NNLO calculations [3, 4, 5] due to the sizeable constant term related to $11.01+[37.58]_{c_{3}, n_{f}}$ in (3.2). Contrary to the NNLO approximation the third-order result shows good convergence of the perturbative expansion. The bottom panel of Figure 1 consequently displays a strong reduction of the scale dependence from NNLO to NNNLO. The residual scale dependence is similar at NNNLO and in the renormalization-group improved calculations, which already captures correctly the logarithms of $\mu$. It therefore appears that with a complete NNNLO 



Figure 1: (top) Successive approximations to the $R$-ratio at fixed $\mu=30 \mathrm{GeV}$. At NNNLO two implementations of $c_{3}$ are shown as discussed in the text. (bottom) Renormalization scale dependence at NNLO and NNNLO. Here all known terms in $c_{3}$ are included.

result and a summation of higher-order logarithms at hand, the demands on an accurate theoretical prediction of the cross section near threshold can be met as far as QCD corrections are concerned. In particular, the scale dependence of the peak position which is indicative of the accuracy of the top mass measurement is now well below $100 \mathrm{MeV}$.

\section{Summary}

The NNNLO QCD correction to the $t \bar{t}$ cross section near threshold is now nearly complete. We presented for the first time the result of the third-order potential and ultrasoft correction to the non-relativistic heavy-quark correlation function. We find that the third-order correction behaves well (contrary to the anomalously large effect at NNLO) and removes a large part of the theoretical uncertainty. The new non-logarithmic terms are numerically important and increase the cross section relative to the renormalization-group improved NNLO result by about $10 \%$, when all presently known terms of the three-loop matching coefficient $c_{3}$ are included. Further work is necessary on a consistent treatment of electroweak and finite-width effects (see [19, 20, 21]). 


\section{Acknowledgments}

This work was supported by the DFG Sonderforschungsbereich/Transregio 9 "Computergestützte Theoretische Teilchenphysik" and DFG Graduiertenkolleg "Elementarteilchenphysik an der TeV-Skala".

\section{References}

[1] A. H. Hoang et al., Eur. Phys. J. direct C 2 (2000) 1 [hep-ph/0001286].

[2] M. Beneke, A. Signer and V. A. Smirnov, Phys. Lett. B 454 (1999) 137 [hep-ph/9903260].

[3] A. H. Hoang, A. V. Manohar, I. W. Stewart and T. Teubner, Phys. Rev. D 65 (2002) 014014 [hep-ph/0107144].

[4] A. H. Hoang, Acta Phys. Polon. B 34 (2003) 4491 [hep-ph/0310301].

[5] A. Pineda and A. Signer, Nucl. Phys. B 762 (2007) 67 [hep-ph/0607239].

[6] M. Beneke, Y. Kiyo and K. Schuller, Phys. Lett. B 658 (2008) 222, arXiv:0705.4518 [hep-ph].

[7] M. Beneke, Y. Kiyo and A. A. Penin, Phys. Lett. B 653 (2007) 53, arXiv:0706.2733 [hep-ph].

[8] M. Beneke, Y. Kiyo, A. Penin and K. Schuller, in preparation.

[9] M. Beneke, Y. Kiyo, A. Penin and K. Schuller, in: Proceedings of 2007 International Linear Collider Workshop (LCWS07 and ILC07), Hamburg, Germany, 30 May - 3 Jun 2007, arXiv:0710.4236 [hep-ph].

[10] V.S. Fadin and V.A. Khoze, Pis'ma Zh. Eksp. Teor. Fiz. 46, 417 (1987) [JETP Lett. 46, 525 (1987)]; Yad. Fiz. 48, 487 (1988) [Sov. J. Nucl. Phys. 48(2), 309 (1988)].

[11] M. Beneke, Y. Kiyo and K. Schuller, Nucl. Phys. B 714 (2005) 67 [hep-ph/0501289].

[12] B.A. Kniehl and A.A. Penin, Nucl. Phys. B 577 (2000) 197.

[13] A.V. Manohar and I.W. Stewart, Phys. Rev. D 63 (2001) 054004.

[14] B.A. Kniehl, A.A. Penin, V.A. Smirnov, and M. Steinhauser, Phys. Rev. Lett. 90 (2003) 212001; Erratum ibid. 91 (2003) 139903.

[15] A.H. Hoang, Phys. Rev. D 69 (2004) 034009.

[16] F. A. Chishtie and V. Elias, Phys. Lett. B 521 (2001) 434 [hep-ph/0107052].

[17] P. Marquard, J. H. Piclum, D. Seidel and M. Steinhauser, Nucl. Phys. B 758 (2006) 144 [hep-ph/0607168].

[18] M. Beneke, Phys. Lett. B 434 (1998) 115 [hep-ph/9804241].

[19] M. Beneke, A. P. Chapovsky, A. Signer and G. Zanderighi, Phys. Rev. Lett. 93 (2004) 011602 [hep-ph/0312331]; Nucl. Phys. B 686 (2004) 205 [hep-ph/0401002].

[20] A. H. Hoang and C. J. Reisser, Phys. Rev. D 71 (2005) 074022 [hep-ph/0412258].

[21] M. Beneke, P. Falgari, C. Schwinn, A. Signer and G. Zanderighi, Nucl. Phys. B 792 (2008) 89 arXiv:0707.0773 [hep-ph]. 